\title{
Numerical studies of elastomeric isolator performance under static loads
}

\author{
Oleg Mkrtychev* \\ Moscow State University of Civil Engineering, Yaroslavskoe shosse, 26, Moscow, 129337, Russia
}

\begin{abstract}
The article presents the studies of the elastomeric isolator performance under static loads. The solutions of the problems have been obtained by means of a specialized software complex by direct integration of the motion equations according to the implicit scheme. For calculation of the elastomeric isolator, a 3D computational model comprised by solid finite elements has been used.

Based on the results of the studies performed, the diagrams for displacement of the points of the top of the support have been plotted with the horizontal shear of the support. The possibility of formation of tilting in the upper plate of the elastomeric isolator has been proved.

The diagram of residual horizontal displacements of the support after the horizontal load removal has been obtained. The inability of elastomeric isolators to return independently to the initial equilibrium position without additional devices has been proved.
\end{abstract}

To assess the effectiveness and appropriateness of using a seismic isolation system in the form of elastomeric isolators (SI) [1,2] in the design of buildings and structures, the respective design-basis justification is required to be accomplished. Different variants of RMO modeling can be used, which are associated with the choice of the element (axial or solid finite elements) and the material describing its performance [3, 4].

In order to simplify the computational model and reduce the calculation time, the SI is usually simulated by an axial finite element, but this option does not fully reflect the actual performance of the support, namely:

- additional sag of the support in case of its shear and impossibility to return to its initial equilibrium position after the horizontal load removal are not taken into account;

- the axial finite element does not allow taking into account the possible tilting (skew) of the support when it is loaded.

The current practice of designing buildings and structures does not take into account these singularities of the seismic isolation. To confirm the above effects we will perform the necessary study of the elastomeric isolator performance under static loads.

For calculation of the elastomeric isolator, a 3D computational model comprised by solid finite elements has been used. The geometric parameters of the support have been taken from the catalogues of the elastomeric isolators manufacturers [5]:

- support diameter: $680 \mathrm{~mm}$;

\footnotetext{
* Corresponding author: a_bunov@mail.ru
} 
- rubber layer height: $5 \mathrm{~mm}$; number of layers: 20;

- metal plate layer height: $2 \mathrm{~mm}$; number of layers: 19.

General view and cross section of the finite element computational model of the support are shown in Fig. 1.

a)

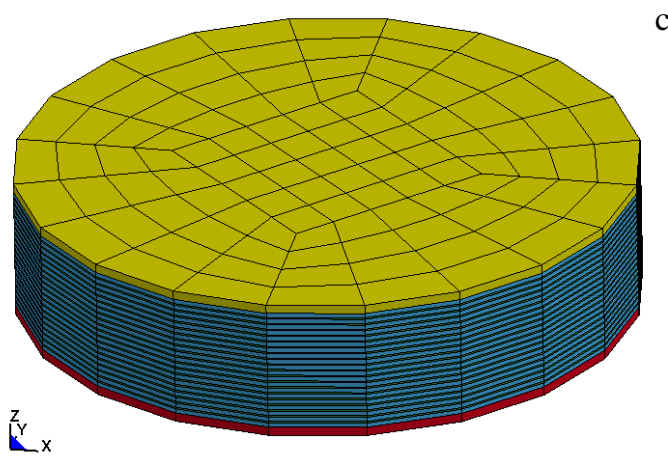

c)

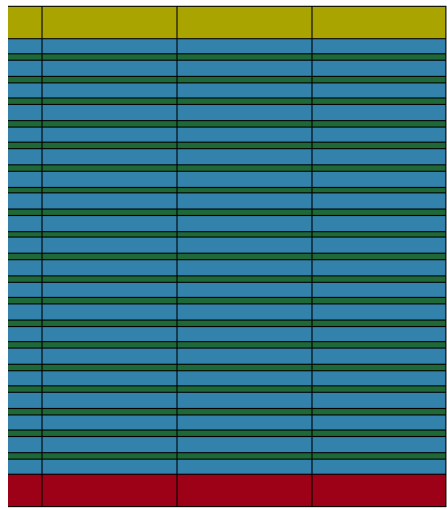

b)

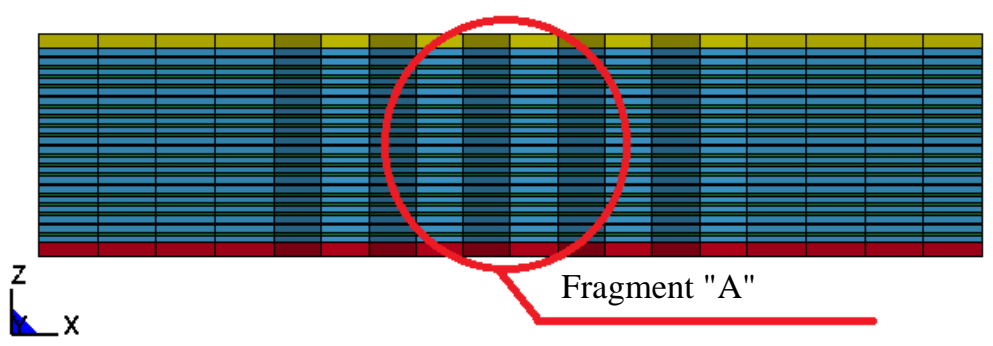

Fig. 1. Elastomeric isolators: a) General view of the finite element computational model of the support; b) Cross section on the body of the finite element computational model of the support; c) Fragment "A" from figure $b$ )

For the rubber material, the following characteristics have been taken:

- shear modulus: 1.6 MPa;

- equivalent viscous damping: $10 \%$;

- density: $1200 \mathrm{~kg} / \mathrm{m} 3$;

- poisson's ratio: 0.49 .

The material of metal sheets: S245 steel.

For the support a maximum value of vertical load $\mathrm{V}=6230 \mathrm{kN}$, and the maximum value of horizontal load $\mathrm{F}=616 \mathrm{kN}$ have been established. Diagrams of external forces acting in the vertical $(\mathrm{Z})$ and horizontal $(\mathrm{X})$ directions are shown in Fig. 2. 
a)

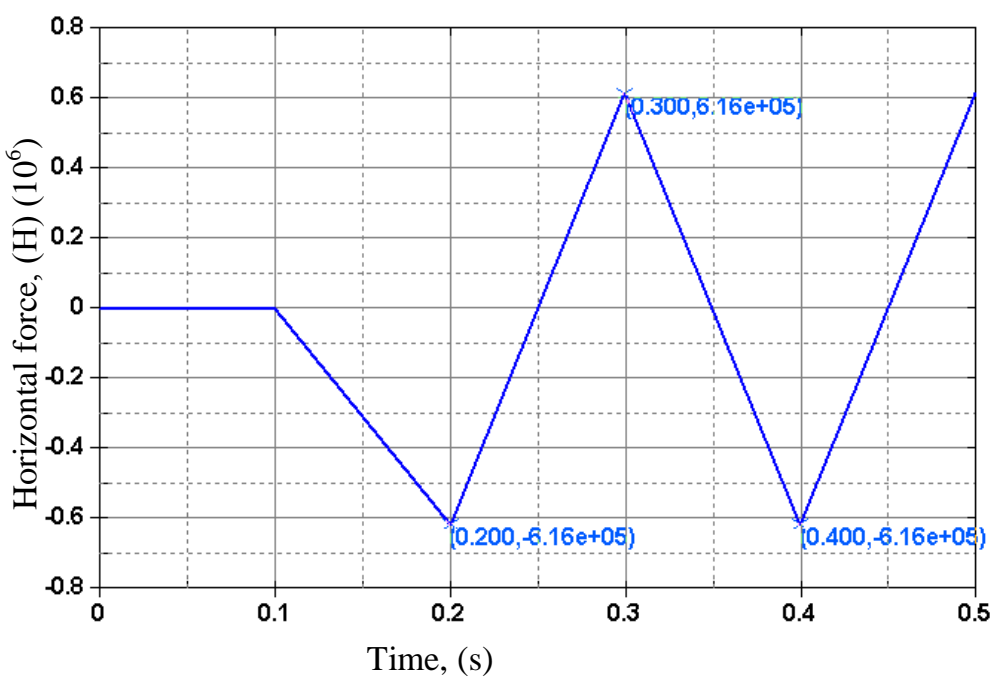

b)

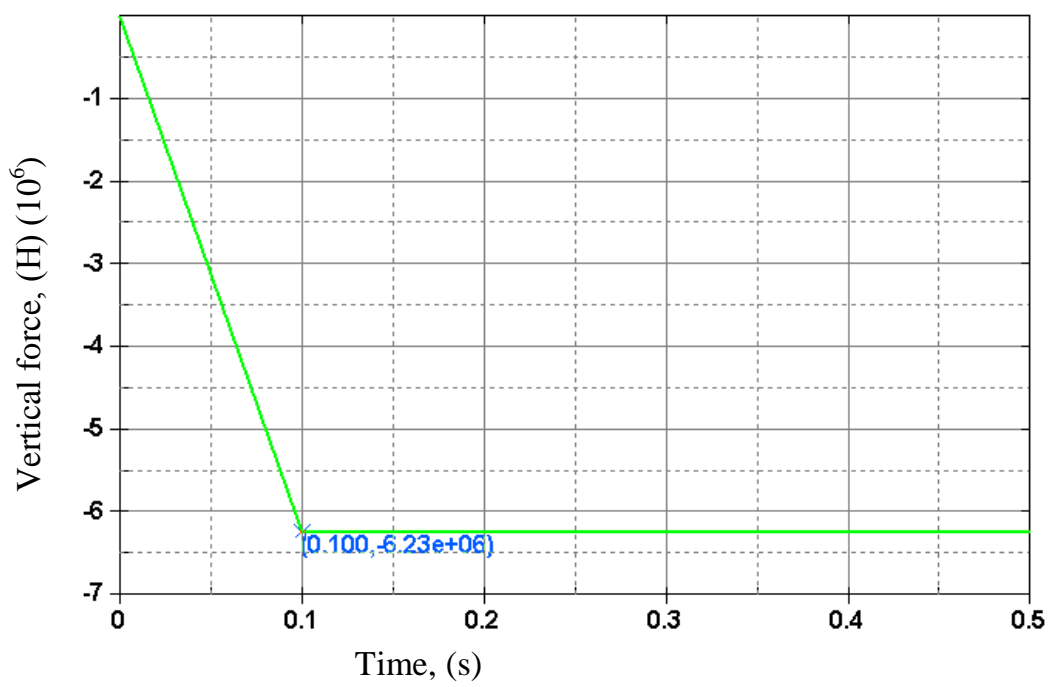

Fig. 2. Diagrams of forces acting: a) the horizontal force in the direction $X$; b) the vertical force in the direction $\mathrm{Z}$

The calculations have been performed using Ansys (LS-Dyna) [6] software taking into account geometrical and physical nonlinearities. The implicit schemes of integration of the motion equations have been used. Below are the main results of the studies performed.

According to the loads calculation data (Fig. 2), a diagram of the elastomeric isolator performance (Fig. 3) has been plotted. 


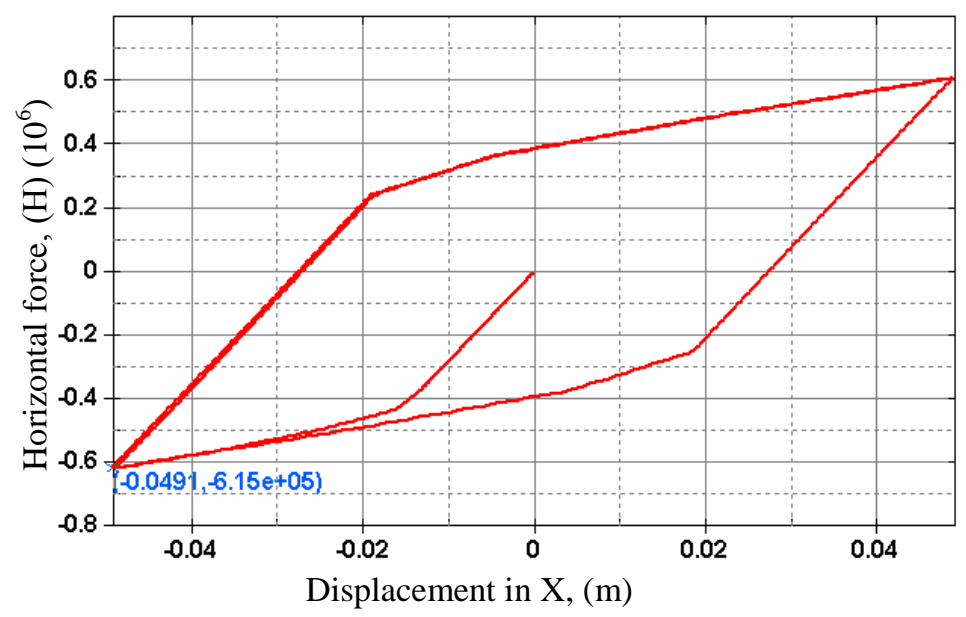

Fig. 3. Diagram of the elastomeric isolator performance

The shape and the basic points of the diagram are coinciding with the data of the elastomeric isolators field testing [5].

To study the presence of internal restoring forces in elastomeric isolators that allow the support to return to its original position upon removal of the horizontal load after deflection, let us set the diagram in Fig. 4 as an initial horizontal (X-direction) force.

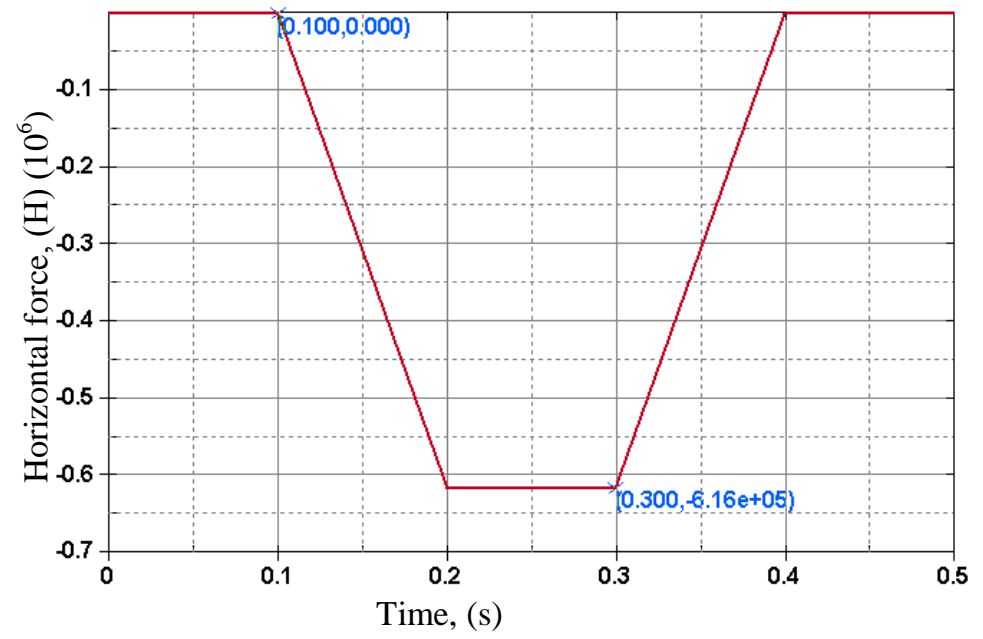

Fig. 4. Diagram of horizontal force in X-direction

According to the calculation results, a diagram of displacement of the points of the top of the support along the X-direction (Fig. 5) has been plotted. 


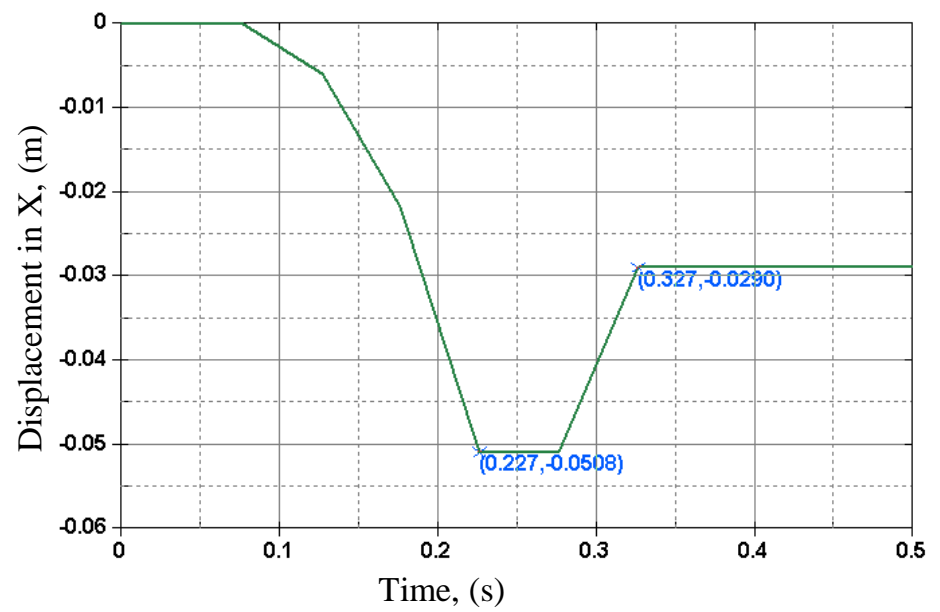

Fig. 5. Diagram of displacement of the points of the top of the support along the X-direction

As it can be seen in Fig. 5, when the horizontal load is removed from the support after its deflection, it does not return to its original position. Internal restoring forces are not enough. A similar conclusion follows from the analysis of experimentally obtained real diagrams of this type of the elastomeric isolator.

Having the same horizontal force (Fig. 4), let us analyze the nature of the vertical displacements of the points of the top of the support. The figures below show the locations of the points and the diagram of their displacements (Fig. 6).

a)

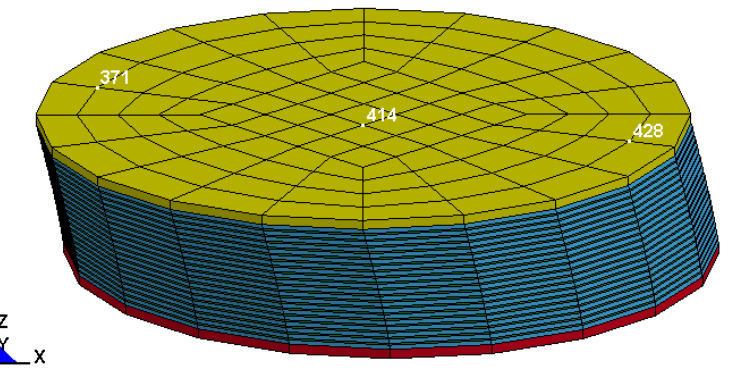

b)



Fig. 6. Vertical displacements of support points: a) Scheme of location of the considered points of the model; b) Graphs of movements of points of support in the $\mathrm{Z}$ direction 
Maximum relative displacements of the points of the top and the bottom of the support, when the top plate of the support is sheared in a horizontal direction under the action of a horizontal force (Fig. 4), are given in Table 1.

Table 1. Maximum relative shear displacement of the points of the top and the bottom of the support

\begin{tabular}{|l|l|l|}
\hline \multirow{2}{*}{ Points of the model } & \multicolumn{2}{|l|}{ Maximum relative shear displacement, $\mathrm{m}$} \\
\cline { 2 - 3 } & Displacement in X & Displacement in Z \\
\hline No 371 & 0,0508 & $-0,00881$ \\
\hline No 414 & 0,0508 & $-0,00683$ \\
\hline No 428 & 0,0508 & $-0,00484$ \\
\hline
\end{tabular}

From the results of Table 1 and Fig. 6 it can be seen that at the support shear in a horizontal direction a part of the top bearing plate sags, and the opposite part is lifted relative to its initial position.

As a result of studies of SI simulated by solid finite elements, the following has been established and confirmed:

1. Shear of the elastomeric isolator results in its additional vertical sag. The simulation of the support by an axial finite element does not take this sag into account.

2. After removing the horizontal load the elastomeric isolators are incapable of returning to their original equilibrium position without additional devices [5].

3. When the elastomeric isolator is loaded, its top bearing plate may be tilted (skewed). The simulation of the support by an axial finite element does not take this tilt into account.

4. Simulation of the elastomeric isolators in software complexes with axial finite elements leads to significant errors in the results of calculations. The contribution of these errors is possible to be assessed only after additional computational studies and verification of the axial finite element model based on the results of experiments or more adequate models using bulk elements and materials that allow to take into account the nonlinear nature of the deformation of the SI.

This study was performed with the financial support of the RF Ministry of Education and Science, grant \#7.1524.2017/Project Part.

\section{References}

1. Trevor E.Kelly, R.Ivan Skinner, Bill Robinson, Ist ed., Kanpur:NICEE, pp. 41-87 (2010)

2. N.P. Murav'ev, Far East: Problems of Development of the Architectural and Construction Complex, 1, pp. 212-218 (2013)

3. A. Mathai, M.S. Manasa, International Journal of Science Technology \& Engineering, 3, pp. 33-36 (2017)

4. R.N. Guzeev, V.A. Petrov, K.Ju. Polinkevich, Natural and technogenic risks. Security of buildings, 5, pp. 41-43 (2013)

5. Catalogue on elastomeric isolators series SI, FIP Industriale S.P.A. (2018)

6. LS-DYNA, Keyword user's manual, I, II (2018) 